\title{
CERTAIN TOPOLOGICAL GROUPS ARE TYPE I ${ }^{1}$
}

\author{
BY ROBERT R. KALLMAN
}

Communicated by Gian-Carlo Rota, September 22, 1969

The purpose of this paper is to prove a theorem which roughly states that if $G$ is a topological group with a "sufficiently large" Type I subgroup $H$, then $G$ itself is also Type I. The main tool in the proof of this theorem is a result of Tomiyama [3] which gives a sufficient condition for a subalgebra of a Type I von Neumann algebra to be Type I. As far as the author knows, this result of Tomiyama has not been applied previously to group representations.

The main result is the following theorem. We note that in the special case in which $G$ and $H$ are separable locally compact groups, $H$ is a closed normal subgroup of $G$, and $G / H$ is compact, the following theorem may be deduced from Mackey [1].

Theorem 1. Let $G$ be a topological group and let $H$ be a closed subgroup of $G$. Suppose that $G / H$ is locally compact and possesses a nonzero, finite, regular, G-invariant Borel measure. Then $G$ is Type I if $H$ is Type I.

Using methods first developed by Sakai [2], Tomiyama [3] proved the following lemma. It is of fundamental importance in what follows.

Lemma 2. Let $\boldsymbol{R}$ and $\mathbf{S}$ be von Neumann algebras with $\mathbf{S} \subseteq R$. Suppose that there exists a linear mapping $P: R \rightarrow S$ with the following properties:

(1) $P(I)=I$;

(2) $P(A) \geqq 0$ if $A \geqq 0$;

(3) $P(A B C)=A P(B) C$ ( $A$ and $C$ in $\mathrm{S}, B$ in $R$ );

(4) $P$ is continuous in the ultraweak operator topology. Then $S$ is Type I if $R$ is Type I.

In what follows, let $\pi: G \rightarrow G / H$ be the natural quotient mapping.

Let $U$ be a strongly continuous unitary representation of $G$ on the Hilbert space $K$. Let $R(G)$ (respectively, $R(H)$ ) be the von Neumann algebra on $K$ generated by $[U(a) \mid a$ in $G]$ (respectively, by $[U(a) \mid a$ in $H]$ ). We have that $R(H) \subseteq R(G) . R(H)$, and therefore $R(H)^{\prime}$, is Type I by assumption.

AMS Subject Classifications. Primary 4665.

Key Words and Phrases. von Neumann algebras, operator theory.

1 Research was supported in part by NSF Grant GP-9141. 
For $a$ in $G$, let $\phi(a)$ be the *-automorphism of $L(K)$ defined by $\phi(a)(T)=U(a) T U(a)^{*}$. We have that $a \rightarrow\langle\phi(a)(T) x, y\rangle$ is continuous for all $T$ in $L(K)$ and $x, y$ in $K$. Note that if $T$ is in $R(H)^{\prime}, \phi(a)(T)$ $=\phi(b)(T)$ in case $a$ and $b$ belong to the same $G / H$ coset; for if $b$ $=a h(h$ in $H)$, then $\phi(b)(T)=U(a) U(h) T U(h)^{*} U(a)^{*}=U(a) T U(a)^{*}$ $=\phi(a)(T)$. Hence, the $\phi(a)$ induce a collection $\psi(m)(m$ in $G / H)$ of *-homomorphisms of $R(H)^{\prime}$ into $L(K)$ by $\psi(m)(T)=\phi(a)(T)(\pi(a)$ $=m, T$ in $\left.R(H)^{\prime}\right)$. The mapping $m \rightarrow\langle\psi(m)(T) x, y\rangle\left(T\right.$ in $R(H)^{\prime}$ and $x, y$ in $K)$ is continuous on $G / H$ since $a \rightarrow\langle\phi(a)(T) x, y\rangle$ is continuous and $\pi: G \rightarrow G / H$ is open.

Let $\mu$ be the nonzero, finite, regular, $G$-invariant, Borel measure on $G / H$. Normalize $\mu$ so that $\mu(G / H)=1$. For $T$ in $R(H)^{\prime}$, let $P(T)$ $=\int_{G / H} \psi(m)(T) d \mu(m)$ (a weak integral). It is clear that $P$ is a linear mapping on $R(H)^{\prime}$. $P$ has its range in $R(G)^{\prime}$, for

$$
\begin{aligned}
\phi(a)(P(T)) & =\int_{G / H} \psi(a \cdot m)(T) d \mu(m) \\
& =\int_{G / H} \psi(m)(T) d \mu(m) \quad \text { ( } \mu \text { is left invariant). }
\end{aligned}
$$

We next verify that $P$ satisfies the conditions (1)-(4) of Lemma 2. (1) and (2) are easy to check. (3) holds since

$$
\begin{aligned}
\psi(m)(A B C) & =\psi(m)(A) \psi(m)(B) \psi(m)(C) \\
& =A \psi(m)(B) C,
\end{aligned}
$$

for all $A$ and $C$ in $R(G)^{\prime}$ and $B$ in $R(H)^{\prime}$.

To check (4), we must show that if $\left[T_{\alpha}\right]$ is an increasing directed net of positive elements of the unit ball of $R(H)^{\prime}$ which converges in the weak operator topology to $T$, then $P\left(T_{\alpha}\right)$ converges to $P(T)$ in the weak operator topology.

Let $x$ be a unit vector in $H$. Given $\epsilon>0$, choose a compact subset $C$ of $G / H$ such that $\mu(G / H-C) \leqq \epsilon$. $C$ exists since $\mu$ is regular. Now $\left\langle\psi(m)\left(T_{\alpha}\right) x, x\right\rangle \uparrow\langle\psi(m)(T) x, x\rangle$ for each $m$ in $C$. But $\left\langle\psi(m)\left(T_{\alpha}\right) x, x\right\rangle$ and $\langle\psi(m)(T) x, x\rangle$ are continuous functions of $m$. Hence, by Dini's theorem, there exists an index $\beta$ such that $\alpha \geqq \beta$ implies

$$
\sup _{m \in C}\left[\langle\psi(m)(T) x, x\rangle-\left\langle\psi(m)\left(T_{\alpha}\right) x, x\right\rangle\right] \leqq \epsilon .
$$

Hence, easy estimates show that $\alpha \geqq \beta$ implies

$$
\langle P(T) x, x\rangle-\left\langle P\left(T_{\alpha}\right) x, x\right\rangle \leqq 3 \epsilon .
$$


Hence, $P$ is continuous in the ultraweak operator topology. The conditions (1)-(4) of Lemma 2 are satisfied. Hence, Lemma 2 implies that $R(G)^{\prime}$ is Type I. Hence, $R(G)$ is Type I. Q.E.D.

\section{BiBLIOGRAPHY}

1. G. W. Mackey, Unitary representations of group extensions. I, Acta Math. 99 (1958), 265-311. MR 20 \#4789.

2. S. Sakai, On topological properties of $W^{*}$-algebras, Proc. Japan Acad. 33 (1957), 439-444. MR 20 \#5437.

3. J. Tomiyama, On the projection of norm one in $W^{*}$-algebras. III, Tôhoku Math. J. (2) 11 (1959), 125-129. MR 21 \#6547.

Massachusetts Institute of Technology, Cambridge, Massachusetts 02139 